\title{
The Research of Automatic Inverted Garage Car Based on STM32
}

\author{
Huang Jian \\ XiJing University, Xi'an 710123, China; \\ 565200245@qq.com
}

Keywords: Intelligent vehicle;STM32; MPU6050;Ultrasonic ranging

\begin{abstract}
In recent years, smart vehicles have become the focus of research. Unmanned vehicles have been developed successfully, and automatic reverse lane and automatic side parking are part of the functions. With several sensors combined with high speed microprocessor, automatic reverse library function can be realized. 3 ultrasonic ranging sensor, combined with the rotation angle measurement of MPU6050 car, the car parking information to make accurate judgments, in order to control the car forward, backward, turn left, turn right, realize automatic reversing function library.
\end{abstract}

\section{Introduction}

In recent years, smart vehicle is a research hotspot. The intelligent vehicle includes automatic driving, automatic reverse storage and automatic side parking. Integrating a variety of sensor technology, including camera acquisition, graphic image recognition, radar technology, GPS navigation technology, and many other high-tech, is an important direction of future development. The problem of automatic parking and side parking is a problem that the intelligent vehicle must solve. Automatic reverse library, to accurately determine the location of the garage, and distance measurement. Accurate control of the car to the target garage. Software programming should be carried out to control the driving of the car, so as to achieve the function of automatic down library.

\section{Hardware Design}

\section{Motor Drive Circuit Design}

The motor drive circuit is shown in figure 1. The PWMA, AIN2 and AIN1 control a motor, in which the PWMA controls the speed of the motor, and the direction of the motor is controlled by the AIN2 and AIN1. PWMB, BIN2 and BIN1 control another motor, in which the PWMB controls the speed of the motor, and the direction of the motor is controlled by BIN2 and BIN1. A TB6612 implementation of the 2 motor control, two TB6612 realize the control of 4 motors, to control the car is 4 drive or 2 drive.

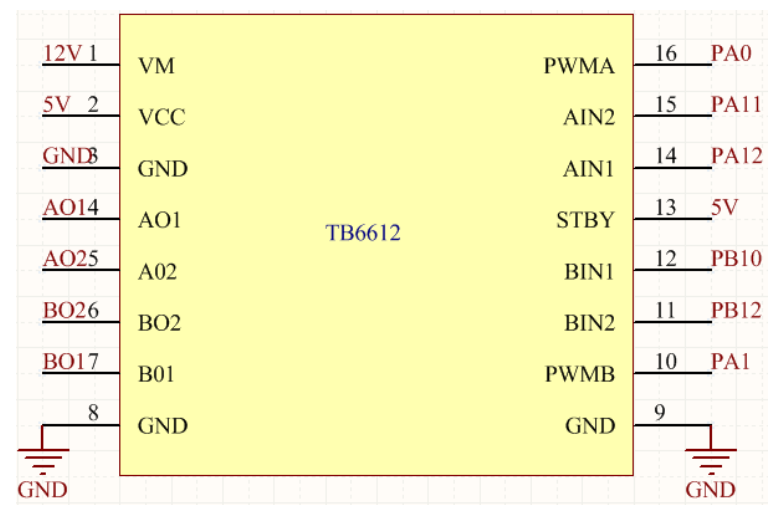

Figure 1 TB6612 motor drive circuit diagram

\section{Design of Ultrasonic Distance Measuring Circuit}

The ultrasonic ranging circuit is shown in figure 2 . The diagram uses $+5 \mathrm{~V}$ power supply, and OUT is the ultrasonic data output end. It can connect any serial port of STM32 and receive data. 


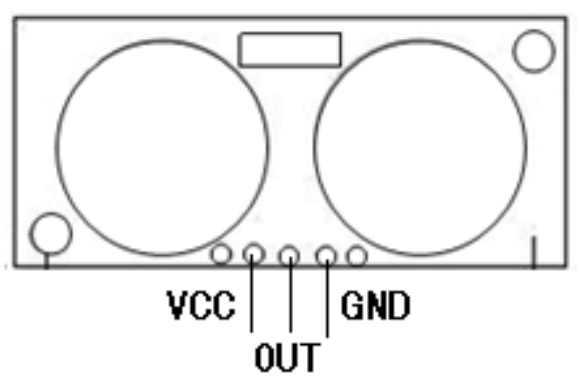

Figure 2 ultrasonic ranging circuit

\section{Software Program}

In this design, under KEIL 5, programming with $\mathrm{C}$ language. Complete ultrasonic ranging, motor control, MPU6050 angle measurement and so on. Some code is given below:

void PD2_Count(void)

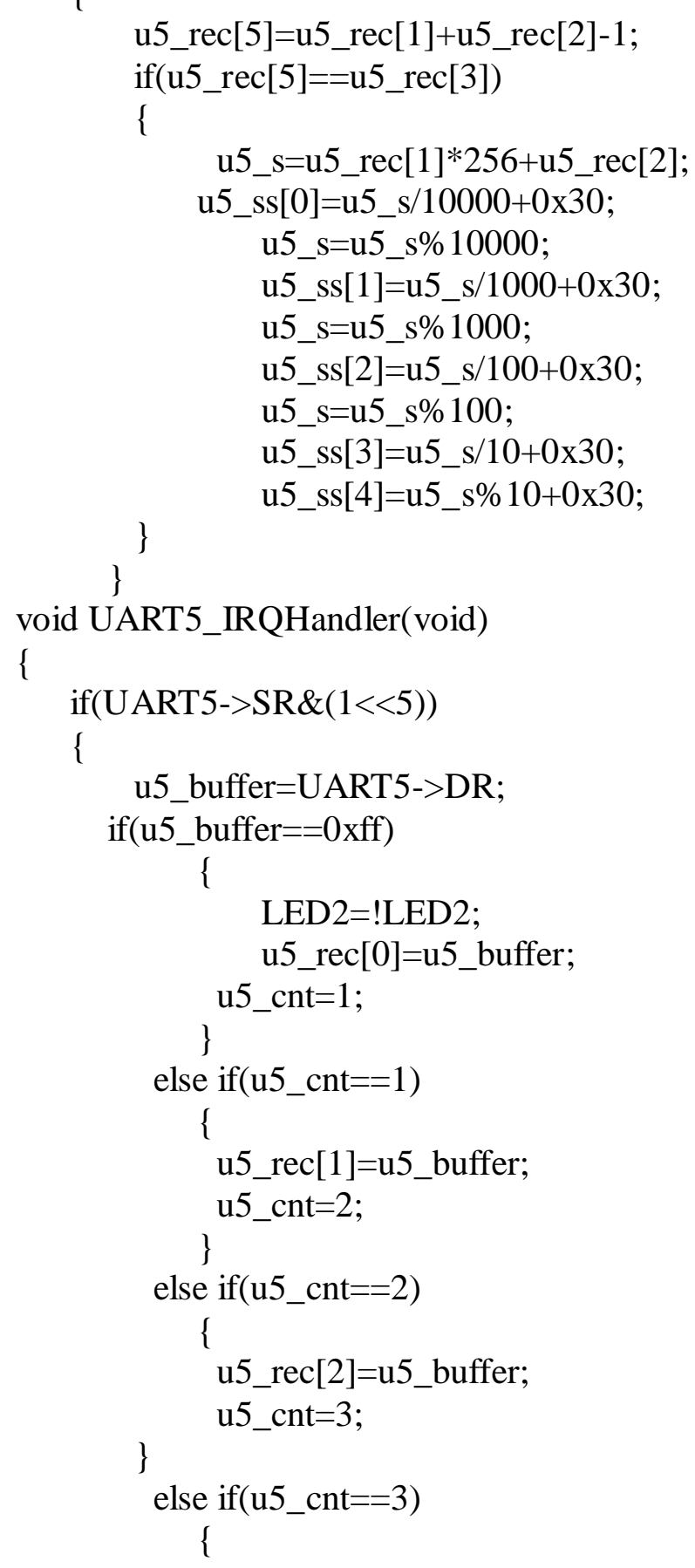




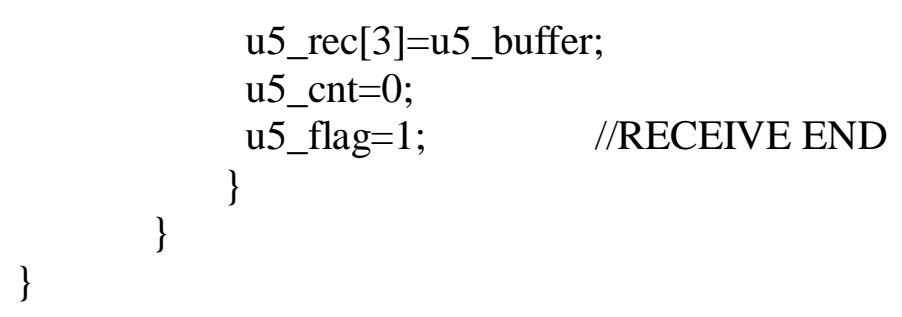

\section{Summary}

This paper expounds the realization method of automatic dumping car, using MPU6050 sensor, ultrasonic ranging module and so on. The design idea, the hardware circuit design and software programming are carried out, and the location of the garage is identified. And use software programming to calculate the front, back, left and right position of the car, and control the driving of the car by TB6612, and realize the function of automatic reverse library, which has certain practical value.

\section{Reference}

[1] Zhang Dongming, Li Xuan. Vertical parking with ultrasonic sensors. Journal of Beijing Union University, 2014,28 (2): 43-47. [J]

[2] TI.LDC1000 inductive digital converter [M]., Shanghai, 2014, 21-23.

[3] Ding Suming, et al. Design and test of DC closed type wind tunnel for.NJS-1 type plant protection [J]. Chinese Journal of agricultural engineering, 2015,31 (4): 76-86.

[4] Lu Fang Cheng. The natural wind conditions insulator contamination characteristics of [J]. high voltage technology wind tunnel simulation, 2014:40 (5): 1281-1289.

[5] Shi Yanping, Liu Chengwen, Ni Lixue. Dynamic track of amorphous alloy pressing magnetic research on the inductive stress sensor based on Journal of sensor technology[J].2009,22 (12): 1704-1708. 